\title{
Medical Treatment for Microscopic Colitis: A Community Hospital's Experience
}

\author{
Abdallah Haidar ${ }^{\mathrm{a}, \mathrm{d}}$, Savreet Kaur ${ }^{\mathrm{a}}$, Nancy Jackson ${ }^{\mathrm{b}}$, Jose Mari Parungao ${ }^{\mathrm{c}}$, \\ Michael Piper ${ }^{\mathrm{c}}$, Alan Cutler ${ }^{\mathrm{C}}$
}

\begin{abstract}
Background: Lymphocytic colitis (LC) is a chronic disorder characterized by watery diarrhea. This study sought to evaluate if LC recurs after therapy, and the time frame in which this occurs. Secondary objectives included length and type of therapy, drug-free intervals, and reasons for drug discontinuation.
\end{abstract}

Methods: A retrospective chart review between January 1, 2008 and October 30, 2015 of patients with biopsy-confirmed lymphocytic, collagenous, or microscopic colitis was conducted. Patient-reported average bowel movements/day were reviewed. Demographic data, dates of colonoscopy, follow-up, type and dose of medications used, and therapy start/stop dates were reviewed.

Results: Patients presenting with colonoscopic documented LC (n $=114$ ) were predominantly female $(88 \%)$, Caucasian $(97 \%)$, with mean bowel movements/day of five. A total of 58/114 (51\%) patients were placed on a therapy. Patients taking budesonide saw bowel movements/day reduced from 4.7 to 2.4 compared to 5.8 to 2.8 for those given 5-aminosalicylic acid (5-ASA). First-line medications budesonide and 5-aminosalicylic acid failed in 12/58 (21\%) of patients, other drugs also resulted in therapy changes. Thirty-five percent required their initial therapy changed and of those $40 \%$ required a second change. Symptom exacerbations were documented during therapy for $19 \%$ of patients; therapy changes resulted in good response.

Conclusions: Almost half of all LC cases (56/114) gradually improved without requiring therapy. Seventy-six percent of our treated patients responded well to budesonide when used as the first-line therapy. Similarly, $61.5 \%$ responded to 5-ASA. Budesonide was the

Manuscript submitted September 29, 2017, accepted October 27, 2017

aDepartment of Internal Medicine, Providence Hospital and Medical Centers, Southfield, MI, USA

bDepartment of Research, Providence Hospital and Medical Centers, Southfield, MI, USA

'Division of Gastroenterology, Providence Hospital and Medical Centers, Southfield, MI, USA

${ }^{\mathrm{d} C o r r e s p o n d i n g ~ A u t h o r: ~ A b d a l l a h ~ H a i d a r, ~ D e p a r t m e n t ~ o f ~ I n t e r n a l ~ M e d i c i n e, ~}$ Providence Hospital and Medical Centers, 16001 West Nine Mile Rd, Southfield, MI 48075, USA. Email: abdhaidar@gmail.com

doi: https://doi.org/10.14740/gr907w drug of choice for flares. Tailoring drug therapy to meet individual patient's needs appears to be the best current approach to managing LC.

Keywords: Lymphocytic colitis; Collagenous colitis; Microscopic colitis

\section{Introduction}

Lymphocytic colitis (LC) and collagenous colitis (CC) are chronic disorders that share similar symptom patterns characterized by watery diarrhea, and they are collectively referred to as microscopic colitis (MC) [1, 2]. In addition to diarrhea, patients may have abdominal pain, weight loss and fecal incontinence. Patients with $\mathrm{MC}$ will have normal colonic mucosa under direct visualization; however, when viewed under microscopy, an abnormal and characteristically histopathological pattern is seen. MC has a yearly incidence of about $5-10$ per 100,000 worldwide. The etiology for MC is not very well understood. However, associations with autoimmune diseases as well as use of non-steroidal anti-inflammatory drugs (NSAIDs) and proton pumps inhibitors (PPIs) have been described [1,2]. The clinical course for $\mathrm{MC}$ is usually benign, although there have been reported cases of mortality [1]. The incidence and prevalence of $\mathrm{MC}$ is on the rise [1,2], where about $10-20 \%$ of all cases of non-bloody watery diarrhea can be attributed to MC [2]. Multiple regimens have been utilized for treatment, including antidiarrheal and anti-inflammatory medications (steroids, immunosuppressant). Recurrence, longer durations of treatment, and long term follow-up are not very well studied. Therefore, the aim of this study was to evaluate if lymphocytic colitis (LC) recurs after therapy, and the time frame in which this occurs. Secondary objectives included evaluation of length and type of therapy, drug-free intervals, if any, the length of these intervals, and the possible clinical reasons for drug discontinuation.

\section{Patients and Methods}

Data from patients with "CC", "LC" or "MC", confirmed by histology between January 1, 2008 and October 30, 2015, were retrospectively identified and supplemented by chart review 
Table 1. Demographics of Patients With Biopsy Confirmed Lymphocytic, Collagenous or Microscopic Colitis

\begin{tabular}{llll}
\hline & ALL & Treated & Post-therapy 1 \\
\hline Number & 114 & $51 \%(58 / 114)$ & - \\
Type & & & - \\
$\quad$ CC & $77 \%(88 / 114)$ & $81 \%(47 / 58)$ & - \\
$\quad$ LC & $16 \%(18 / 114)$ & $15 \%(9 / 58)$ & - \\
$\quad$ MC & $7 \%(8 / 114)$ & $3 \%(2 / 58)$ & - \\
Race/Caucasian & $97 \%(113 / 114)$ & - & - \\
Gender/female & $88 \%(111 / 114)$ & $86 \%(50 / 58)$ & - \\
Average age & $69 \pm 13$ & $69 \pm 11$ & - \\
Associated autoimmune diseases $(\mathrm{RA} \mathrm{n}=3, \mathrm{SLE} \mathrm{n}=2$, psoriasis & $7 \%(8 / 114)$ & - & \\
$\mathrm{n}=1$, Hashimoto's thyroiditis $\mathrm{n}=1$, temporal arteritis $\mathrm{n}=1)$ & & & \\
Average BMs/D & $5.0 \pm 3.3$ & $5.1 \pm 3.4$ & $2.9 \pm 2.4$ \\
\hline
\end{tabular}

CC: collagenous colitis; LC: Iymphocytic colitis; MC: microscopic colitis; RA: rheumatoid arthritis; SLE: systemic lupus erythematosus; BMs/D: bowel movements per day.

from our community hospital and its outpatient gastroenterology clinics. Index cases were identified and retrieved through electronic medical records via a data warehouse request employing the International Classification of Diseases, 9th Revision (ICD-9) codes associated with this disease spectrum. Average daily number of bowel movements as reported by the patients was reviewed at specific time points: pre-treatment (or on the initial encounter), at baseline (during therapy) and during exacerbations. While a definition for clinical remission has been proposed, and is currently defined as a mean of $<$ 3 stools/day or a mean of $<1$ watery stool/day, the opposite may be used to define recurrence: a mean of $\geq 3$ stools/day or a mean of $\geq 1$ watery stool/day [1]. Demographic data including age, race, and gender were included. Date of colonoscopy and dates of follow-up were gathered. Start and stop dates of therapy were reviewed. Medication type and dosage were also gathered. Electronic and hard copy data including test results, demographic information, and clinic visit notes and medical records were the main sources of information.

Data source included medical records, pathology results, colonoscopy results and clinic visit notes. The Material was recorded on a data-sheet anonymously with no code numbers to link the research data to the subject identifiers.

Inclusion criteria were 1) patients $\geq 18$ years old, 2) patients who had colonoscopy with biopsies, 3) patients diagnosed with $\mathrm{MC}$, LC or CC based on histologically confirmed tissue biopsy, 4) patients who were treated for $\mathrm{LC}, \mathrm{CC}$ or $\mathrm{MC}$, and 5) patients who have clinical recurrence of $\mathrm{LC}, \mathrm{CC}$ or $\mathrm{MC}$ confirmed by diarrhea, defined as a mean of $\geq 3$ stools/day or a mean of $\geq 1$ watery stool/day.

Exclusion criteria were 1) diagnosis of LC, CC or MC that was not confirmed histologically, and 2) patients with LC, CC or $\mathrm{MC}$ who did not receive treatment were excluded from statistical analysis comparing response to therapy.

Microsoft Excel was used for descriptive statistics for all variables. These include means for continuous variables and frequencies for categorical variables. The main outcome variables were LC recurrence, time to recurrence, and the relation- ship of these variables to therapy. The Student's $t$-test (paired and unpaired) was used to compare means.

\section{Results}

From 2008 to 2015 , a total of 114 patients had biopsy-confirmed collagenous $(77 \%)$, lymphocytic $(16 \%)$ or microscopic colitis (7\%). Among these, $88 \%$ were females, and the overall average age at diagnosis was $69 \pm 13$ years, and $69 \pm 11$ years for those who were treated. Of those documented, $97 \%$ were Caucasian and 7\% (8/114) had associated autoimmune disease. Average bowel movements per day (BMs/D) was 5.0 \pm 3.3 at presentation, $5.1 \pm 3.4$ for those who were treated and $2.9 \pm 2.4$ after first therapy (Table 1 ).

A total of 58 patients $(51 \%)$ were documented to be on a therapy with various starting dates (Table 2). Among those, half of the patients were started on oral steroid budesonide, mainly a dose of $9 \mathrm{mg}$ daily with a gradual taper for $\geq 2$ months; oral steroid prednisone was also used but only on a few occasions (Table 3 ). The second most common treatment used was anti-inflammatory drug 5-amino salicylic acid (5-ASA) in $22 \%$ of the patients, with variable daily doses ranging between 1,200 up to $4,800 \mathrm{mg}$. Other less commonly used medications included diphenoxylate/atropine, bismuth subsalicylate, hyoscyamine, cholestyramine, and metronidazole alone or with ciprofloxacin, and lastly fiber tablets. In this study, first-line (budesonide) and second-line (5-ASA) treatments have been shown to be effective with improvement/resolution of diarrhea

Table 2. Patients Starting on Therapy

\begin{tabular}{ll}
\hline Patients on therapy & $\mathbf{5 8}$ \\
\hline Unknown start date & 4 \\
Therapy started prior to colonoscopy & 13 \\
Therapy started on day of colonoscopy & 8 \\
Therapy started after colonoscopy & 33 \\
\hline
\end{tabular}


Table 3. Patients on Therapy, Type, Change and Reason for Change

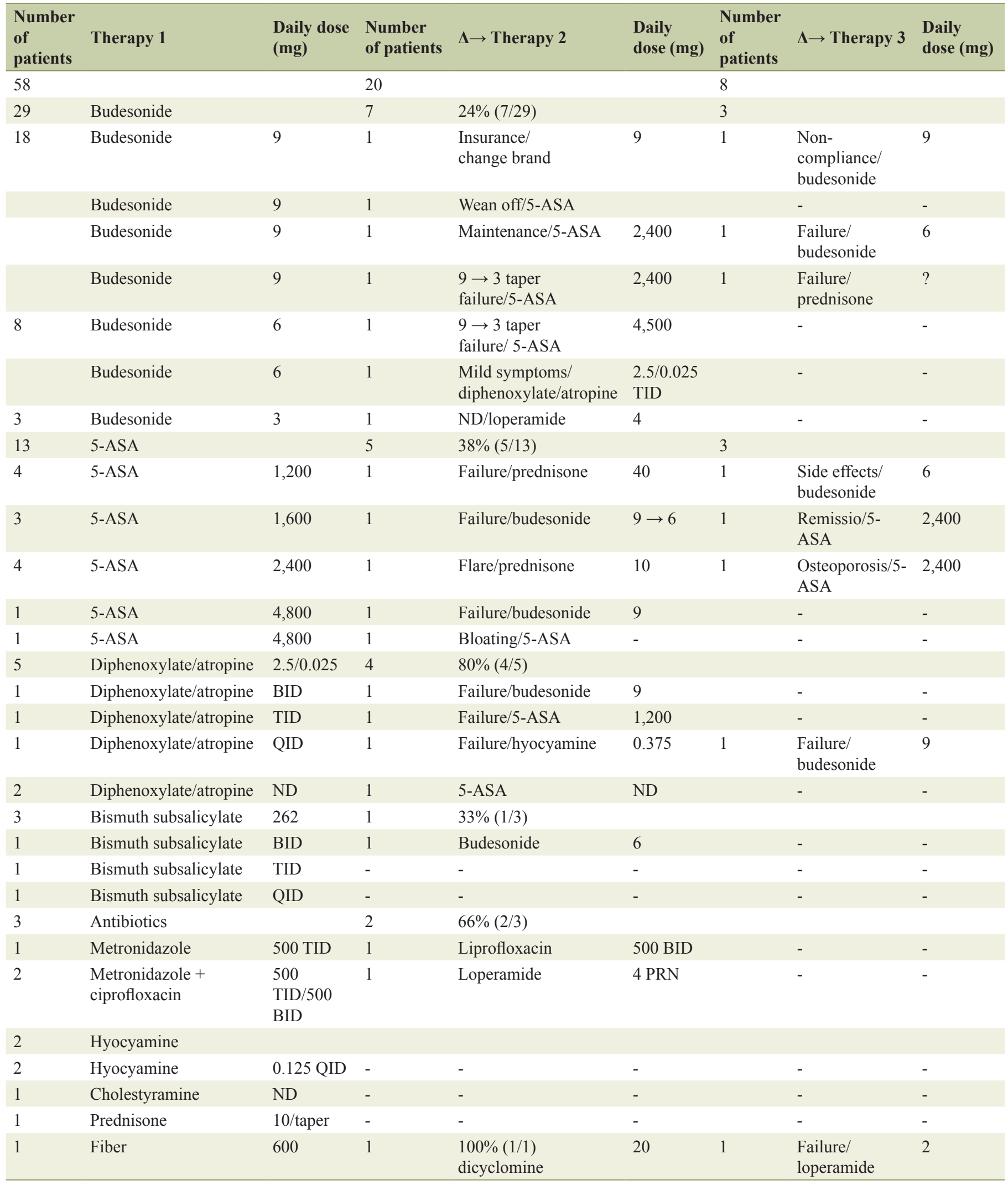

BID: twice daily; TID: three times daily; QID: four times daily; ND: not documented; PRN: as needed. 
Table 4. Average Reduction of Bowel Movements per Day for Budesonide and 5-ASA

\begin{tabular}{lll} 
Therapy & Budesonide & 5-ASA (Mesalamine) \\
\hline $\mathrm{BMs} / \mathrm{D}$ reduced $\rightarrow$ & $4.7 \rightarrow 2.4$ & $5.8 \rightarrow 2.8$ \\
\hline
\end{tabular}

$\mathrm{BMs} / \mathrm{D}$ : bowel movements per day.

in $76 \%(22 / 29)$ and $61.5 \%(8 / 13)$ of the cases, respectively. The remaining $16 / 58$ patients $(27.5 \%)$ had variable responses at follow-up when treated with second line treatments. In $50 \%(8 / 16)$ of patients who used a third line therapy including cholestyramine, bismuth salicylate, hyoscyamine, diphenoxylate-atropine, and even antibiotics including metronidazole alone or with ciprofloxacin or when prednisone was used, the diarrhea resolved without the need for a therapy change (Table $3)$. One third of the patients (20/58) who had therapy 1 , ended up requiring a therapy change into another medication (therapy 2). Reasons to change therapy included, most commonly failure of therapy, in addition to side effects, medical insurance and tapering off in case of budesonide. Similarly, there was a need for a third therapy change in $40 \%(8 / 20)$ patients due to therapy 2 failure, medication noncompliance, and an attempt to switch away from budesonide in order to avoid potential side effects (Table 3 ).

The average duration of treatment was 73 days for the first therapy and 70 days for the second therapy. Of the 29 patients who switched drugs, 20 had no lag time between drugs. The remaining nine patients had an average drug-free interval between therapies of 437 days. Overall, budesonide gave a $49 \%$ decrease in average BMs/D compared to 5-ASA which gave a $52 \%$ decrease (Table 4 ).

Symptom exacerbations were documented during therapy in $11 / 58(19 \%)$ of patients. Four patients were on 5-ASA and were subsequently switched to budesonide $(\mathrm{n}=3)$ and prednisone $(n=1)$. Of note, one of these four patients was treated successfully with 5-ASA $1.6 \mathrm{~g}$ three times daily (TID) for $>21$ months until it was replaced with budesonide due to symptom exacerbation. Three of the patients with symptom flares had been switched from budesonide $9 \mathrm{mg}$ /day to 5-ASA as a flare up was documented after completing $\geq 2$ months of budesonide $9 \mathrm{mg}$ /day: right after discontinuation, after 5 months and after 30 months, respectively. Two patients were on budesonide taper 9 to $3 \mathrm{mg}$ when they had a flare, and one patient was on a low starting dose of budesonide with $3 \mathrm{mg}$ /day used. Lastly, a discontinuation of budesonide $9 \mathrm{mg}$ after 1-month taper use also caused a flare up. Alternating between 5-ASA and budesonide or increasing the initial budesonide dose and/ or prolonging its duration of treatment resulted in symptom improvement or resolution (Table 5).

\section{Discussion}

Our study suggests that lymphocytic, collagenous and microscopic colitis can be treated effectively with the first-line agent oral budesonide with a starting dose of $9 \mathrm{mg}$ /day for at least 8 weeks. This has been also supported in other studies [3-7]. A lower dose of $6 \mathrm{mg}$ /day was effective in only (5/8) $62 \%$ in this study with two of the documented symptom exacerbations occurring upon tapering from 9 to 6 to $3 \mathrm{mg}$, and one therapy change due to failure. When compared to other studies, oral budesonide $6 \mathrm{mg} /$ day was suggested to be efficacious for longterm maintenance of clinical remission in patients with CC [6]. A lower starting dose of $3 \mathrm{mg}$ may fail to control the symptoms. Tapering off budesonide over $\geq 2$ months appears to be more effective than rapid tapering over 4 weeks due to the number of symptoms exacerbations. On the other hand, a duration of treatment of longer than 2 months can be decided based on each individual case, knowing that about $17 \%$ of patients (one out of six) had symptoms exacerbations right after discontinuation of budesonide when it was given for at least 2 months. Overall, and due to the risk of side effects on long term use, budesonide could possibly be tapered off within 2 - 3 months, with an equal expected efficacy (76\%) upon restarting when there are symptom exacerbations. Budesonide, however, might not even be needed as slightly more than half of the patients $(52 \%)$ had their diarrheal symptoms resolved without the use of budesonide. In these cases, second-line agent (5-ASA) with a lower efficacy than budesonide of $61 \%$, which was also supported by other studies [8]. Even third-line agents including cholestyramine, bismuth, hyoscyamine and/or diphenoxylateatropine with up to $50 \%$ efficacy can be tried for controlling milder symptoms. This suggests that the nature of the colitis disease may vary largely among patients who are exposed to different triggering factors and/or have various underlying comorbid conditions.

In conclusion, almost half of all microscopic colitis cases diagnosed by colonoscopy improve without requiring therapy. Patients requiring therapy usually respond well to budesonide. This therapy worked for $76 \%$ of our first line budesonidetreated patients, whereas $61.5 \%$ of our 5 -ASA treated patients responded well to that first line of therapy. Budesonide was the drug of choice for flares, typically $9 \mathrm{mg}$ /day for at least

Table 5. Symptoms Exacerbation Ratios, Therapies Used Before and After Exacerbations

\begin{tabular}{lll}
\hline Symptom exacerbation & $\mathbf{1 1 / 5 8}(\mathbf{1 9 \%})$ & Improved/resolved \\
\hline While on 5-ASA & $(3 / 11)$ & Change to budesonide $9 \mathrm{mg} / \mathrm{day}$ \\
& $(1 / 11)$ & Change to prednisone $10 \mathrm{mg} / \mathrm{day}$ \\
\hline While on budesonide $9 \mathrm{mg}$ /day & $(3 / 11)$ & Change to 5-ASA \\
Lowering budesonide $9 \mathrm{mg}$ to $3 \mathrm{mg}$ & $(2 / 11)$ & Increase budesonide to $9 \mathrm{mg}$ \\
While on budesonide $3 \mathrm{mg} / \mathrm{day}$ & $(1 / 11)$ & Increase budesonide to $9 \mathrm{mg}$ \\
\hline Discontinuation of budesonide $9 \mathrm{mg} /$ day (given for $1 \mathrm{month})$ & $(1 / 11)$ & Restarted budesonide $6 \mathrm{mg} / \mathrm{day} \times 1 \mathrm{month}$, then $3 \mathrm{mg} / \mathrm{day}$ \\
\hline
\end{tabular}


2 months. Tailoring drug therapy to meet individual patient's needs appears to be the best current approach to manage LC.

\section{Author Contributions}

Abdallah Haidar: study design, data collection, statistical analysis, data interpretation, manuscript preparation, literature search and funds collection. Savreet Kaur: study design and data collection. Nancy Jackson: study design, statistical analysis, data interpretation, manuscript preparation and funds collection. Jose Mari Parungao: study design and data interpretation. Michael Piper: study design, data interpretation and manuscript preparation. Alan Cutler: study design, data interpretation and manuscript preparation.

\section{Conflict of Interest}

Abdallah Haidar: None. Savreet Kaur: None. Nancy Jackson: None. Jose Mari Parungao: None. Michael Piper: None. Alan Cutler is a consultant at pharmaceutical Otsuka and Allergan.

\section{References}

1. Farrukh A, Mayberry JF. Microscopic colitis: a review. Colorectal Dis. 2014;16(12):957-964.

2. Brown WR, Tayal S. Microscopic colitis. A review. J Dig
Dis. 2013;14(6):277-281.

3. Bohr J, Wickbom A, Hegedus A, Nyhlin N, Hultgren Hornquist E, Tysk C. Diagnosis and management of microscopic colitis: current perspectives. Clin Exp Gastroenterol. 2014;7:273-284.

4. Gentile NM, Abdalla AA, Khanna S, Smyrk TC, Tremaine WJ, Faubion WA, Kammer PP, et al. Outcomes of patients with microscopic colitis treated with corticosteroids: a population-based study. Am J Gastroenterol. 2013;108(2):256-259.

5. Miehlke S, Madisch A, Voss C, Morgner A, Heymer P, Kuhlisch E, Bethke B, et al. Long-term follow-up of collagenous colitis after induction of clinical remission with budesonide. Aliment Pharmacol Ther. 2005;22(1112):1115-1119.

6. Miehlke S, Madisch A, Bethke B, Morgner A, Kuhlisch E, Henker C, Vogel G, et al. Oral budesonide for maintenance treatment of collagenous colitis: a randomized, double-blind, placebo-controlled trial. Gastroenterology. 2008;135(5):1510-1516.

7. Miehlke S, Madisch A, Karimi D, Wonschik S, Kuhlisch E, Beckmann R, Morgner A, et al. Budesonide is effective in treating lymphocytic colitis: a randomized double-blind placebo-controlled study. Gastroenterology. 2009;136(7):2092-2100.

8. Miehlke S, Madisch A, Kupcinskas L, Petrauskas D, Bohm G, Marks HJ, Neumeyer M, et al. Budesonide is more effective than mesalamine or placebo in shortterm treatment of collagenous colitis. Gastroenterology. 